\begin{tabular}{|c|c|}
\hline Title & A nalysis of a synchronously mode locked and internally frequency-doubled CW dye laser \\
\hline Author(s) & Y amashita, Mikio; Y amada, Keita; Sato, Takuzo \\
\hline Citation & $\begin{array}{l}\text { IEEE Journal of Quantum Electronics, 18(1), 95-100 } \\
\text { https://doi.org/10.1109/JQ E.1982.1071383 }\end{array}$ \\
\hline Issue Date & $1982-01$ \\
\hline Doc URL & http:/hdl.handle.net/2115/45312 \\
\hline Rights & $\begin{array}{l}\text { C } 1981 \text { IEEE. Personal use of this material is permitted. However, permission to reprint/republish this material for } \\
\text { advertising or promotional purposes or for creating new collective works for resale or redistribution to servers or lists, } \\
\text { or to reuse any copyrighted component of this work in other works must be obtained from the IEEE. }\end{array}$ \\
\hline Type & article \\
\hline File Information & JQE18-1_95-100.pdf \\
\hline
\end{tabular}

Instructions for use 


\title{
Analysis of a Synchronously Mode-Locked and Internally Frequency-Doubled CW Dye Laser
}

\author{
MIKIO YAMASHITA, KEITA YAMADA, AND TAKUZO SATO
}

\begin{abstract}
We present analytical studies of a synchronously modelocked and internally frequency-doubled CW (SMLFD) dye laser. The analysis describes the cavity detuning behavior of the pulse duration and the square of its peak intensity (the signal intensity of the autocorrelation trace) in terms of system parameters for different lengths of the intracavity crystal. The results predict the broadening of the pulse duration with the increase of the crystal length even though the condition of the group velocity matching is satisfied. It is shown that the prediction is in good agreement with the experimental results of the SMLFD rhodamine $6 G$ laser with an ADP crystal in the cavity. Furthermore, the variation of the minimum pulse duration at the optimum cavity length as a function of the harmonic convetsion efficiency is derived.
\end{abstract}

Manuscript received June 8, 1981; revised August 25, 1981.

The authors are with the Laser Research Section, Radio- and OptoElectronics Division, Electrotechnical Laboratory, Ibaraki-ken, Japan.

\section{INTRODUCTION}

RECENTLY, a synchronously mode-locked and intraR cavity frequency-doubled CW (SMLFD) dye laser has been operated to produce efficiently continuous trains of UV picosecond pulses [1] , [2], and employed for the study of fast photochemical reactions of molecules [3]. In the previous papers [1], [2], it has been briefly discussed that the broadening of the pulse duration of the fundamental wave is mainly due to the following two causes even though the condition of the group velocity matching is satisfied: one is the nonlinear losses due to the frequency-doubling process in the cavity and the linear insertion losses of a nonlinear crystal; the other is the limitation of the intracavity bandwidth due to the birefringent effect of the crystal. The subject of this paper is to analyze quantitatively the characteristics of such a SMLFD dye 
laser, where the effects of the intracavity crystal for secondharmonic generation (SHG) on the duration of the dye laser pulse are described in detail.

The pulse characteristics of the usual synchronously modelocked CW (SML) dye laser were theoretically considered by some authors. Kim et al. [4] presented an analysis on the SML laser in the time domain by the self-consistent approach describing the steady-state pulse properties in terms of the system parameters. In the analysis it was shown that the pulse duration is determined by $\frac{1}{3}$ power of the duration of pumping pulses and inverse $\frac{2}{3}$ power of the bandwidth of a frequency tuning element. Ausschnitt et al. [5] also discussed analytically the effect of cavity length detuning by slightly modifying the Haus' steady-state equation [6] for the dye pulse envelope. They showed that the pulse duration is proportional to $\frac{1}{2}$ power of the duration of pumping pulses and inverse $\frac{1}{2}$ of the bandwidth of the tuning element. The latter model, however; does not reproduce well the experimental result of the broadening of the pulse duration in the region where the cavity length is shorter than the optimum position [7]. Our analysis is performed with an exterision of the former model so as to include the effects of the nonlinear losses due to the SHG process, the birefringent filter due to the intracavity SHG crystal, and the insertion losses of the crystal.

An intracavity SHG in a forced mode-locked Nd:YAG laser by a loss modulator was analyzed by Falk [8]. In the analysis it was quantitatively shown that the envelope of the electric field with fundamental frequency emerging from the SHG crystal suffers decrease in the amplitude and broadening in the pulse duration, while essentially maintaining the original pulse envelope. This result is applied to the effect of the SHG process on the dye pulse envelope in the SMLFD dye laser.

In the next section, the basic analytical model is briefly reviewed and fundamental equations describing the characteristics of the SMLFD dye laser are presented, while in Section III their solutions are derived. Section IV gives cavity detuning characteristics of the pulse duration and the square of its peak intensity for different lengths of the intracavity crystal, and the variation of the pulse duration as a function of the conversion efficiency of SHG, relevant to the experimental results of the SMLFD rhodamine $6 G$ laser with an intracavity ADP crystal.

\section{Fundamentals of the ANALYsis}

The normalized envelope $V(t)$ of the electric field amplitude of the dye pulse is assumed to be Gaussian, and the propagation of its pulse is traced around the optical cavity shown schematically in Fig. 1. The effect of each optical component on the Gaussian pulse is considered, and it is required that pulse envelope repeats itself after a roundtrip traversal of the cavity.

The analysis makes the following assumptions: 1) for a homogeneously broadened two-level dye laser system, both pulse envelopes of the pumping ion laser and the dye laser are Gaussian; 2) the lifetime of the upper level of dye molecules is much longer than the duration $2 \sqrt{\ln 2} t_{p}$ of the pumping pulse but is shorter than the cavity roundtrip time; 3 ) the gain profile

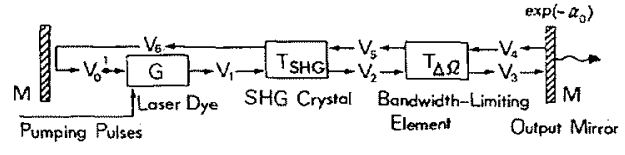

Fig. 1. Schematic of the synchronously mode-locked and internally frequency-doubled CW (SMLFD) dye laser.

is much broader than the pulse bandwidth; 4) the effect of the multiple pass through the frequency-doubling crystal is to alter the Gaussian pulse parameters such as the duration $\tau_{p}$ and the amplitude $V(t)$, while maintaining a Gaussian envelope. This is generally thought to be reasonable, since the practical conversion efficiency of SHG is less than one percent [8].

The temporal variation of the dye-pulse envelope at point 1 next to the laser medium in Fig. 1 is given by

$$
V_{0}(t)=\exp \left(-\gamma t^{2}\right)
$$

where the duration $\tau_{p}$ of the pulse intensity is equal to $[2 \ln 2 /$ $\gamma]^{1 / 2}$. The input signal to the active medium is amplified by the pumping pulse $\Lambda \times\left(1 / \sqrt{\pi t_{p}}\right) \times \exp \left\{-\left[(t-\Delta t) / t_{p}\right]^{2}\right\}$ of the duration $2 \sqrt{\ln 2} t_{p}$ and the pump energy $\Lambda$ absorbed in its medium per unit volume. The $\Delta t=2\left(L_{p}-L_{d}\right) / c$ is the time lag between pump and dye pulse peaks corresponding to the difference between pump $L_{p}$ and dye $L_{d}$ laser cavity-length. After passage through the medium, the pulse envelope becomes

$$
V_{1}(t)=G(t) V_{0}(t)
$$

by using the single-pass gain $G(t)[4]$. The gain at the line center in the case where the duration of the pumping pulse is much longer than that of the dye pulse is expressed by

$$
G(t)=\exp \left(g+\delta \tau^{2}\right) \times \exp \left\{-\delta(t-\tau)^{2}\right\}
$$

in terms of an effective gain factor

$$
g=g_{0} \times \operatorname{erfc}\left(\frac{\Delta t}{t_{p}}\right) \times \exp \left(-\frac{1}{2 T_{s}} \sqrt{\frac{\pi}{2 \gamma}}\right)
$$

a gain-modulation strength

$$
\delta=\frac{2 g_{0}}{\sqrt{\pi} t_{p}} \times \exp \left\{-\left(\frac{\Delta t}{t_{p}}\right)^{2}\right\} \times\left[\exp \left(-\frac{1}{2 T_{s}} \sqrt{\frac{\pi}{2 \gamma}}\right)\right] \times \frac{1}{T_{s}}
$$

and an amount shifted in time of the modulation peak from the peak of the amplified pulse

$$
\tau=\frac{1}{2}\left[T_{s}-\frac{1}{2} \sqrt{\pi} t_{p} \times \operatorname{erfc}\left(\frac{\Delta t}{t_{p}}\right) \times \exp \left(\frac{\Delta t}{t_{p}}\right)^{2}\right] .
$$

The notation used follows [4]: the small signal gain $g_{0}=\Lambda \sigma_{e}$ $l / 2$, the saturation time $T_{s}=\left[c \sigma_{e} E_{0}^{2} / 2 \hbar \omega_{0}\right]^{-1}$, and erfc denotes the complementary error function. The reduction of (2) to a Gaussian form leads to

$$
\begin{aligned}
V_{1}(t) & =\exp \left(g+\frac{\delta^{2} \tau^{2}}{\delta+\gamma}\right) \times \exp \left[-(\delta+\gamma)\left(t-\frac{\delta \tau}{\delta+\gamma}\right)^{2}\right] \\
& \equiv A_{1} \exp \left[-\gamma_{1}\left(t+t_{10}\right)^{2}\right]
\end{aligned}
$$

where 


$$
A_{1} \equiv \exp \left(g+\frac{\delta^{2} \tau^{2}}{\delta+\gamma}\right), \gamma_{1} \equiv \delta+\gamma, \text { and } t_{10} \equiv-\delta \tau /(\delta+\gamma)
$$

Next, the pulse passes through the frequency-doubling crystal. The output pulse resulting from the passage is found by one application of the transfer function of the crystal [8]:

$$
\begin{aligned}
V_{2}(t)= & A_{1} \exp \left(-\sqrt{2} \alpha_{S} / 2-\alpha_{l} / 2\right) \times \exp \left[-\left(1-\sqrt{2} \alpha_{S}\right)\right. \\
& \left.\cdot \gamma_{1}\left(t+t_{10}\right)^{2}\right] \\
\equiv & A_{2} \exp \left[-\gamma_{2}\left(t+t_{10}\right)^{2}\right]
\end{aligned}
$$

where

$$
\begin{aligned}
A_{2} \equiv & \exp \left(g+\frac{\delta^{2} \tau^{2}}{\delta+\gamma}-\frac{\sqrt{2}}{2} \alpha_{S}-\frac{\alpha_{l}}{2}\right) \\
& \text { and } \lambda_{2} \equiv\left(1-\sqrt{2} \alpha_{S}\right)+(\delta+\gamma) .
\end{aligned}
$$

The $\alpha_{S}$ and $\alpha_{l} / 2$ are the conversion efficiency to the SH wavelength and the linear insertion losses of the crystal for the single pass through its crystal, respectively. The process of the pulse broadening due to the SHG may be understood as follows. During the SHG process the power of the fundamental wave is mostly converted to the SH wave at the pulse peak of the fundamental one and is only very slightly converted at its extremes. This is in contrast to the effect of the nonlinear absorption losses due to a saturable dye in the cavity. Consequently, this power-dependent loss to the intracavity propagating pulse leads to substantial broadening of the pulse duration. The transfer function of (8) is a mathematical expression of this effect by a single parameter $\alpha_{S}$, defined as the average power conversion efficiency to the SH wavelength.

When the pulse passes the bandwidth-limiting element having the transfer function $T(\omega)=\exp \left[-D^{\prime}\left(\omega-\omega_{0}\right)^{2}\right]$, the envelope function becomes

$$
V_{3}(t)=\frac{A_{2}}{\sqrt{4 D^{\prime} \gamma_{2}+1}} \exp \left[-\frac{\gamma_{2}}{4 D^{\prime} \gamma_{2}+1}\left(t+t_{10}\right)^{2}\right]
$$

where $D^{\prime} \equiv(2 \sqrt{\ln 2} / \Delta \Omega)^{2}$, and $\Delta \Omega$ is the full bandwidth at half maximum point of the element. Furthermore, the pulse suffers a net. roundtrip loss $\alpha_{0}$ including the output coupling effect, except for the losses relevant to the intracavity crystal, i.e., $V_{4}(t)=\exp \left(-\alpha_{0}\right) V_{3}(t)$.

After the pulse is reflected by the output mirror, it again passes through the bandwidth-limiting element. The output $V_{5}(t)$ is found by one more application of (9). This means that $D^{\prime}$ in (9) must be replaced by $D \equiv 2 D^{\prime}$.

Similarly, $V_{s}(t)$ is the input for the second pass through the frequency-doubling crystal, so that the output-field envelope $V_{6}(t)$ becomes

$$
\begin{aligned}
V_{6}(t)= & \frac{A_{1} \exp \left[-\left(\sqrt{2} \alpha_{S}+\alpha_{i}+\alpha_{0}\right)\right]}{\sqrt{4 D\left(1-\sqrt{2} \alpha_{S}\right) \gamma_{1}+1}} \\
& \times \exp \left[-\frac{\left(1-2 \sqrt{2} \alpha_{S}\right) \gamma_{1}}{4 D\left(1-\sqrt{2} \alpha_{S}\right) \gamma_{1}+1}\left(t+t_{10}\right)^{2}\right] .
\end{aligned}
$$

For self-consistancy, the net modification of the pulse upon return to the starting point must be set equal to zero. That is, the initial input pulse $V_{0}(t)$ must be the same as the final out put pulse $V_{6}(t)$, aside from a phase factor. This leads to three coupled equations by equating powers of $t$

$$
\begin{aligned}
& g+\frac{\delta^{2} \tau^{2}}{\delta+\gamma}-\frac{1}{2} \ln \left[\frac{32 \ln 2}{\Delta \Omega^{2}}\left(1-\sqrt{2} \alpha_{S}\right)(\delta+\gamma)+1\right]-\sqrt{2} \\
& \cdot \alpha_{S}-\alpha_{1}-\alpha_{0}=0 \\
& \gamma=\frac{\left(1-2 \sqrt{2} \alpha_{S}\right)(\delta+\gamma)}{\frac{32 \ln 2}{\Delta \Omega^{2}}\left(1-\sqrt{2} \alpha_{S}\right)(\delta+\gamma)+1} \\
& \Delta L=-\frac{c}{2}\left[\frac{\delta \tau}{\delta+\gamma}\right]
\end{aligned}
$$

where the cavity length detuning $\Delta L \equiv L_{d}-L_{p}$ is equal to $-c \Delta t / 2$. Equations (11), (12), and (13), respectively, represent the conservations of the pulse amplitude, the pulse duration, and the position of the pulse peak through a roundtrip. The fundamental equations provide a complete description of cavity length detuning characteristics of the SMLFD dye laser. That is, these can determine the duration $\tau_{p}=[2 \ln 2 / \gamma]^{1 / 2}$ of the pulse and the square $\left|E_{0}^{2}\right|^{2}$ of its peak intensity as a function of length detuning $\Delta L$ for any SHG conversion efficiency $\alpha_{S}$ and limited bandwidth $\Delta \Omega$ due to the intracavity crystal, as shown in the following section.

\section{Solution to the Equations}

From (4) and the saturation time $T_{s}=\left[c \sigma_{e} E_{0}^{2} / 2 \hbar \omega_{0}\right]^{-1}$, the pulse energy $\epsilon=E_{0}^{2}[2 \ln 2 / \gamma]^{1 / 2}$ is written

$$
\frac{\epsilon}{\epsilon_{S}}=\ln \left[\left(\frac{g_{0}}{g}\right) \times \operatorname{erfc}\left(\frac{\Delta t}{t_{p}}\right)\right]
$$

where $\epsilon_{s}$ is the saturation value [4]. The substitution of (12) and (13) into $\delta$ and $\tau$ in (11) enables us to describe $g$ in (14) in terms of system parameters

$$
g=\alpha+\frac{\left[A_{0} / 2-(\Delta t)^{2}\right] \gamma_{0}}{B_{0}-A_{0} \gamma_{0}}
$$

where the effective total cavity losses $\alpha \equiv \sqrt{2} \alpha_{S}+\alpha_{l}+\alpha_{0}$, the effective bandwidth-limiting factor

$$
A_{0} \equiv 32 \ln 2 \times\left(1-\sqrt{2} \alpha_{S}\right) / \Delta \Omega^{2}
$$

and the pulse broadening factor due to SHG process $B_{0} \equiv$ $1-2 \sqrt{2} \alpha_{S}$. The $\gamma_{0}$ is the positive solution of the quadratic equation for $\gamma$ which is derived from the rearrangement of $(11)$ by substituting (4), (5), and (12)

$$
\begin{aligned}
\gamma_{0}= & \left\{\left[\left(\left(\Delta t+\tau_{0}\right)^{2}-B_{0} \tau_{0}^{2}\right]^{2}+4 \alpha A_{0} B_{0} \tau_{0}^{2}\right\}^{1 / 2}\right. \\
& \left.-\left[\left(\Delta t+\tau_{0}\right)^{2}-B_{0} \tau_{0}^{2}\right]\right\} / 2 A_{0} \tau_{0}^{2}
\end{aligned}
$$

where

$$
\tau_{0} \equiv\left(\sqrt{\pi} t_{p} / 2\right) \times \operatorname{erfc}\left(\Delta t / t_{p}\right) \times \exp \left(\Delta t / t_{p}\right)^{2} .
$$

Here, the third term in in (11) has been expanded to first order, and the term $\left(\alpha-\frac{1}{2}\right) A_{0}$ in $\gamma_{0}$ has been neglected. Equation 
TABLE I

System Parameters Employed for the Calculation Which Werk OBTAINED From THE EXPERIMENT ON AN SMLFD RHODAMINE 6G laser [1] and Calculated Pulse Durations

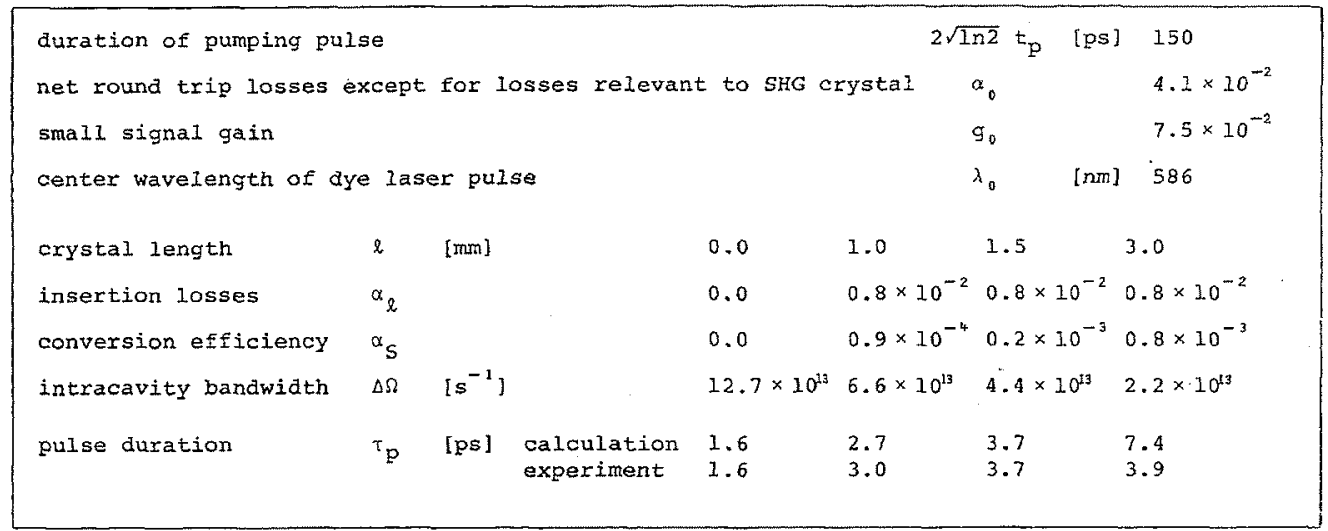

(14) with the use of (15) and (16) enables us to determine the pulse energy of the fundamental wave in the SMLFD dye laser. When the second term in (15) is neglected without the intracavity SHG effect, (14) corresponds to (9) in [4] for the usual SML laser.

Next, we describe the pulse duration of the fundamental wave in terms of the system parameters in the SMLFD dye laser. The substitution of (5) and (14) into one (12) of the fundamental equations yields the cubic equation for $\gamma^{1 / 2}$

$$
A_{0} \gamma^{3 / 2}+A_{0} F \gamma+\left(1-B_{0}\right) \gamma^{1 / 2}-B_{0} F=0
$$

where

$$
F \equiv\left(4 \sqrt{2} g_{0} / \pi t_{p}\right) \times \exp \left\{-\left(\Delta t / t_{p}\right)^{2}\right\} \times \exp \left(-\epsilon / \epsilon_{S}\right) \times \epsilon / \epsilon_{S}
$$

Solving by the Cardan approach [9], we obtain the positive solution

$$
\gamma^{1 / 2}=\alpha^{1 / 3}+\beta^{1 / 3}-\frac{F}{3}
$$

and

$$
\begin{aligned}
a, \beta= & \left\{9\left(1+2 B_{0}\right) F-2 A_{0} F^{3} \pm 3 \sqrt{3 / A_{0}}\left[4\left(1-B_{0}\right)^{3}\right.\right. \\
& \left.\left.-A_{0} F^{2}\left(1-20 B_{0}-8 B_{0}^{2}\right)-4 A_{0}^{2} B_{0} F^{4}\right]^{1 / 2}\right\} / 54 A_{0} .
\end{aligned}
$$

Therefore, the pulse duration $\tau_{p}=[2 \ln 2 / \gamma]^{1 / 2}$ is determined by the use of (18) and (19). If $6 \sqrt{2} \alpha_{S} \gg A_{0} F^{2} \gg \alpha_{S}^{3}$, simplification of (18) and (19) can be made

$$
\gamma^{1 / 2}=\left[\left(2 B_{0}+1\right) F / 3 A_{0}\right]^{1 / 3}
$$

hence, the corresponding pulse duration is

$$
\tau_{p}=(2 \ln 2)^{1 / 2} \times\left[3 A_{0} /\left(2 B_{0}+1\right) F\right]^{1 / 3} .
$$

This reduces to (11) in [4] when $\alpha_{S}=\alpha_{l}=0$.

\section{EFFECTS OF InTRACAVITy SHG CRystal ON Pulse Duration}

The experiment on a SMLFD rhodamine $6 \mathrm{G}$ laser pumped by an Ar ion laser has been carried out by employing an ADP crystal of a different length as a frequency-doubler [1], [2] . For confirmation of the analysis, we compare the experimental results by the use of laser parameters of the system. The relevant parameters are summarized in Table I [1].
Fig. 2 shows the variations of the duration $\tau_{p}$ and the square $\left(\epsilon / \epsilon_{s}\right)^{2} / \tau_{p}^{2}$ of the peak intensity of the fundamental pulse as a function of length detuning $\Delta L$ for different crystal-length $l$. The behavior of the variations explains well the experimental fact that the cavity length where the pulse duration becomes minimum is shorter by several $\mu \mathrm{m}$ than the length where the SH intensity of the autocorrelation signal becomes maximum $[1],[2]$. From Fig. 2 we also find that with increase of the crystal length the pulse duration is broadened, which agrees with the experimental findings. The increase of the crystal length brings about not only the quadratic increase of the nonlinear losses $\alpha_{S}$ due to the SHG process, but also the linear decrease of the intracavity bandwidth $\Delta \Omega$ due to the birefringent effect of the crystal. The bandwidth $\Delta \Omega_{1.5}$ employed in the calculation is adjusted so that both the theoretical and experimental minima of the pulse duration in the case of the $1.5 \mathrm{~mm}$ length $l_{1.5}$ of the ADP crystal coincide. For different crystal lengths, the bandwidth $\Delta \Omega_{l}$ employed is equal to the product $l_{1.5} \cdot \Delta \Omega_{1.5} / l$ between the bandwidth $\Delta \Omega_{1.5}$ for the $1.5 \mathrm{~mm}$ length and the inverse ratio of each crystal length $l$ to the $1.5 \mathrm{~mm}$ length $l_{1.5}$, since the bandwidth is inversely proportional to its length [10]. The calculated values of the minimum pulse duration for different crystal lengths are given in Table I with the ones obtained experimentally [1].

Next, we consider separately, the effect of the nonlinear losses $\alpha_{S}$ corresponding to the practical conversion efficiency of SHG and the bandwidth-limiting effect $\Delta \Omega$ of the crystal on the pulse duration. The calculated dependence of the minimum pulse duration on the conversion efficiency with keeping the intracavity bandwidth constant is shown in Fig. 3. On the other hand, in Fig. 4 the dependence of the pulse duration on the bandwidth while keeping the conversion efficiency constant is shown. From these figures it is established that the pulse duration increases rapidly as the increase of the conversion efficiency is more than $\alpha_{S} \gtrsim 1 \times 10^{-3}$ and as the decrease of the bandwidth is less than $\Delta \Omega \lesssim 2 \times 10^{13} \mathrm{~s}^{-1}$. Therefore, it is deduced that in the present experimental system both the effects of the nonlinear losses due to the SHG process and the intracavity bandwidth-limiting due to the birefringence of the crystal, cause the pulse broadening by nearly the same degree.

All the numerical values, thus far, have been calculated by 


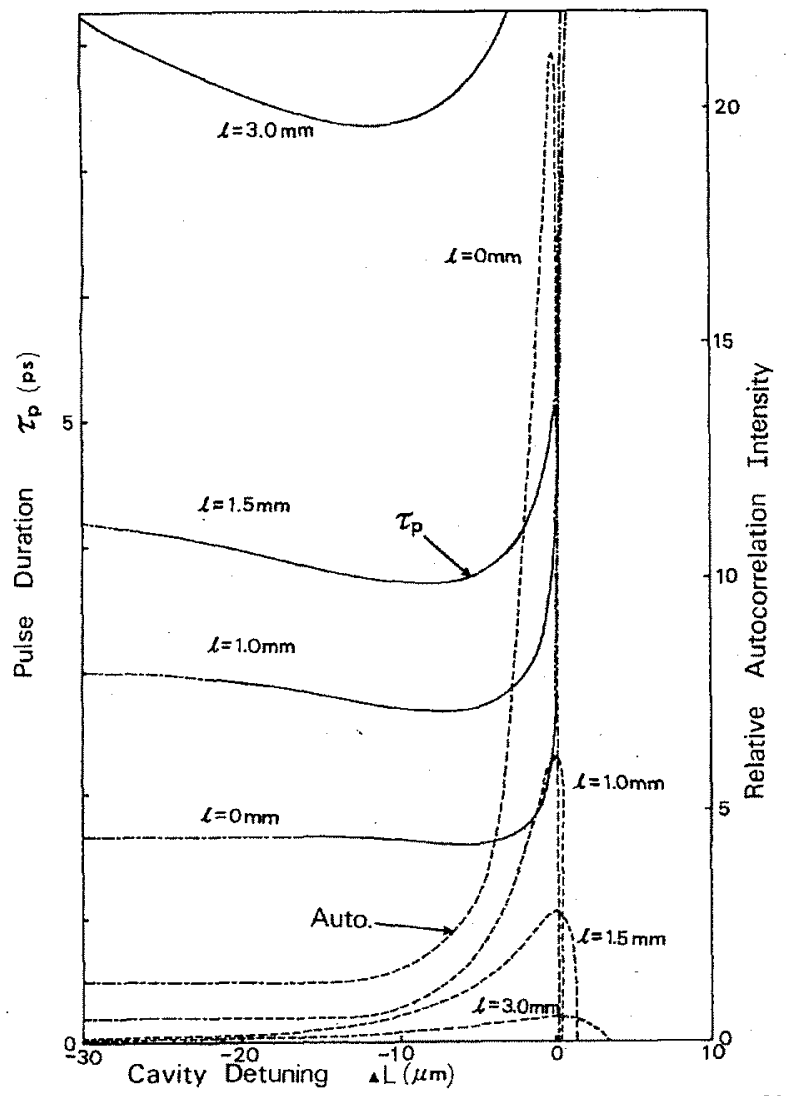

Fig. 2. Variation of the pulse duration (solid lines) and the square of its peak intensity (dashed lines) of the fundamental wave as a function of cavity length detuning for different lengths of the intracavity SHG crystal. The reference point of $\Delta L=0$ is taken at the point of the cavity length where the square of the pulse peak intensity is maximum.

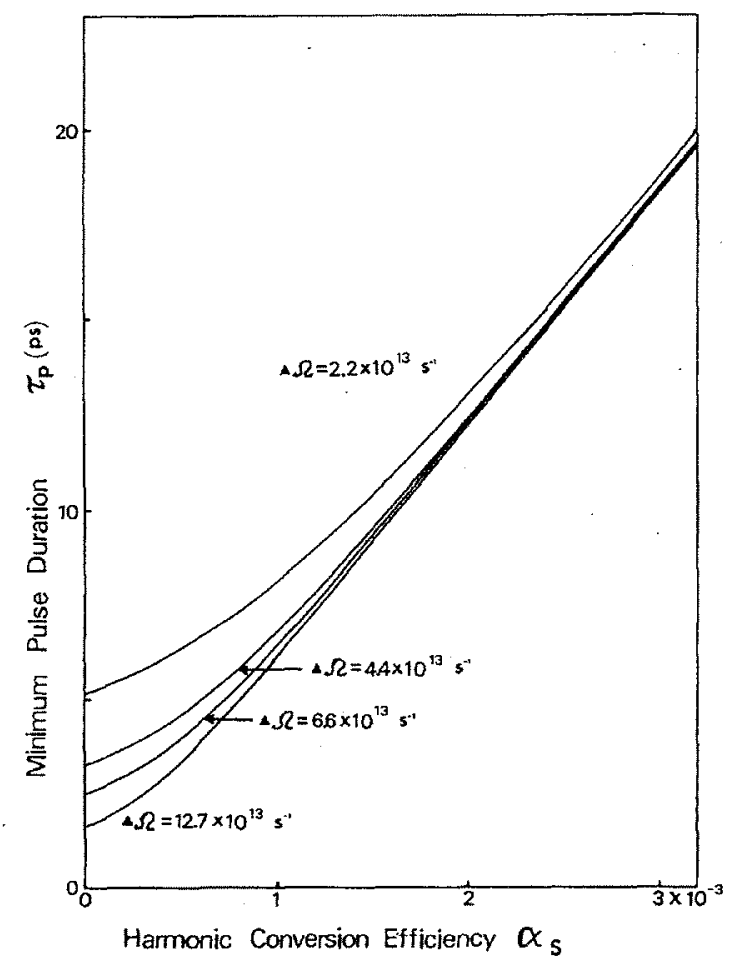

Fig. 3. Dependence of the minimum pulse duration on the SHG conversion efficiency for different intracavity bandwidths.

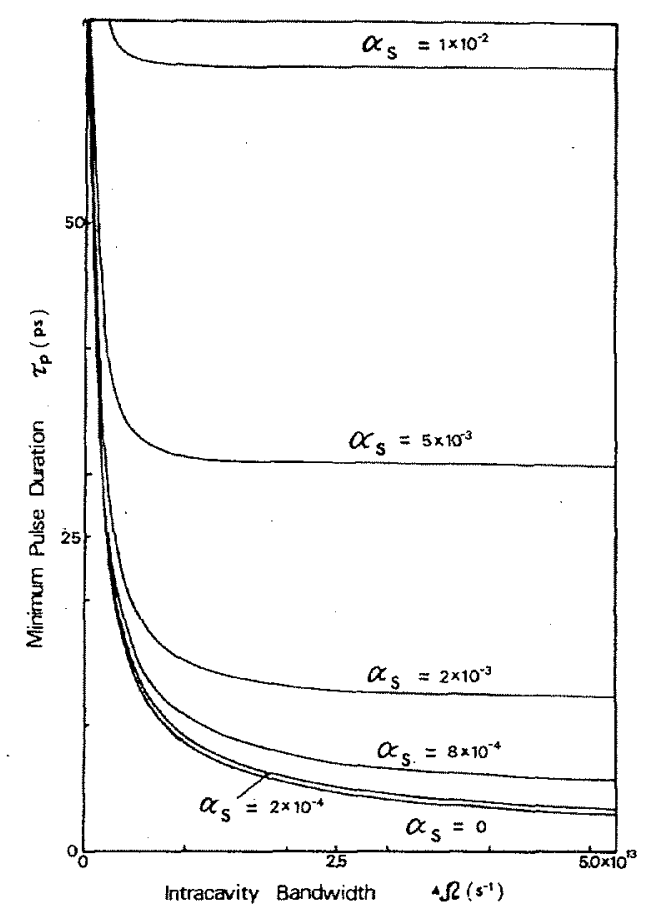

Fig. 4. Dependence of the minimum pulse duration on the intracavity bandwidth for different conversion efficiencies to the SH wavelength.

the use of the exact solution (18) and (19). In order to examine whether or not the approximate solution of $(20)$ is proper, we have also calculated length detuning characteristics for different crystal lengths by the use of its solution. The result has indicated that the approximation is quite reasonable as long as the conversion efficiency is less than $\sim 2 \times 10^{-4}$.

Our model has a limitation. The pulse shape of the dye laser has been assumed to be a Gaussian, unlike the result predicted from the experiment of the SMLFD dye laser [1]. This is a consequence of the application of the approximate solution to the single-pass gain $G(t)$ of the active medium. The $G(t)$ in (3) has been obtained by making Taylor expansion to second order in time about the peak location of the dye pulse since the gain modulation of interest is the change near the peak of $G(t)$ within the duration of its pulse [4], [5]. The expansion is valid only near the pulse peak in the vicinity of the gain peak, but not in the wings. A correct description of pulse-shaping effect would require an exact computer solution of the gain evolution. However, our model is still able to reproduce the cavity detuning behavior for the effect of intracavity SHG in the good mode-locking region in the vicinity of the gain peak.

\section{CONCLUSION}

This paper has analyzed the characteristics of a SMLFD dye laser. For different lengths of the intracavity crystal, the variation of the pulse duration and the square of its peak intensity as a function of cavity length detuning has been derived. Furthermore, the dependence of the pulse duration on the conversion efficiency of SHG has been given. It has been shown that these analytical predictions explain quite well the results obtained from the experiment on a SMLFD rhodamine $6 \mathrm{G}$ laser with ADP crystal in the cavity. 


\section{ACKNOWLEDGMENT}

Some parts of the computer work were performed with the help of T. Katakura and N. Haga.

\section{REFERENCES}

[1] M. Yamashita, W. Sibbett, D. Welford, and D. J. Bradley, "Intracavity second-harmonic generation in a synchronously modelocked CW dye laser," J. Appl. Phys., vol. 51, pp. 3559-3562, July 1980.

[2] D. Welford, W. Sibbett, and J. R. Taylor, "Intracavity second harmonic generation using a synchronously mode-locked CW dye laser," Opt. Commun., vol. 35, pp. 283-286, Nov. 1980.

[3] J. R. Taylor, M. C. Adams, and W. Sibbett, "Time resolved fluorescence of trans-stilbene in the picosecond regime," Appl. Phys. Lett., vol. 35, pp. 590-592, Oct. 1979.

[4] D. M. Kim, J. Kuhl, R. Lambrich, and D. von der Linde, "Characteristics of picosecond pulses generated from synchronously pumped CW dye laser system," Opt. Commun., vol. 27, pp. 123 126 , Oct. 1978

[5] C. P. Ausschnitt, R. K. Jain, and J. P. Heritage, "Cavity length detuning characteristics of the synchronously mode-locked $C W$ dye laser," IEEE J. Quantum Electron., vol. QE-15, pp. 912917, Sept. 1979.

[6] H. A. Haus, "Theory of mode locking with a slow saturable absorber," IEEE J. Quantum Electron., vol. QE-11, pp. 736-746. Sept. 1975 .

[7] F. Minami and K. Era, "Near-infrared subpicosecond pulse generation in a synchronously mode-locked CW dye laser," Opt. Commup., vol. 35, pp. 393-395, Dec. 1980.

[8] J. Falk, "A theory of the mode-locked, internally frequencydoubled laser," IEEE J. Quantum Electron,, vol. QE-11, pp. 21$31, J$ an. 1975.

[9] G. A. Korn and T. M. Korn, Mathematical Handbook for Scientists and Engineers. New York: McGraw-Hill, 1961, p. 23.

[10] F. P. Schäfer, Dye Lasers, 2nd ed., F. P. Schäfer, Ed. Berlin: Springer, 1977, pp. 74-75.

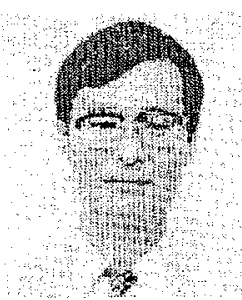

Mikio Yamashita was born in Kyoto, Japan, on February 15, 1946. He received the M.S. and Ph.D. degrees in electronics engineering from Kyoto University, Kyoto, Japan, in 1970 and 1976, respectively.

Since joining the Laser Research Section, Radio- and Opto-Electronics Division, Electrotechnical Laboratory, Tsukuba, Japan, in 1970, he has been engaged with laser excitation ESR studies on laser dyes, development of the tunable dye laser, and its application to the isotope separation. In 1978 he was a Visiting Research Associate at Imperial College. His research interests are currently in the generation of the ultrashort pulse and its application including photochemical biotechnology.

Dr. Yamashita is a member of the Physical Society of Japan, the Japan Society of Applied Physics, and the Laser Society of Japan.

Keita Yamada, photograph and biography not available at the time of publication.

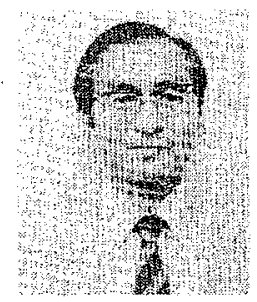

Takuzo Sato was born in Akita, Japan, on October 27, 1940. He received the M.S. and Ph.D. degrees in electronics engineering from Tohoku University, Sendai, Japan, in 1965 and 1980 , respectively.

In 1965 he joined the Electrotechnical Laboratory, Tokyo, Japan, where he has been engaged in research on the $Q$-switched ruby laser dye lasers, mixing of laser beams, laser radar, and excimer lasers. His fields of interest are high-power lasers and their applications.

Dr. Sato is a member of the Institute of Electronics and Communication Engineers of Japan, the Japan Society of Applied Physics, and the Laser Society of Japan. 